\title{
Chaotic domains: A numerical investigation
}

\author{
M. C. Cross, D. Meiron, and Yuhai Tu \\ Condensed Matter Physics and Applied Mathematics, California Institute of Technology, Pasadena, \\ California 91125
}

(Received 29 November 1993; accepted for publication 4 May 1994)

\begin{abstract}
We study the chaotic domain state in rotating convection using a model equation that allows for a continuous range of roll orientations as in the experimental system. Methods are developed for extracting the domain configuration from the resulting patterns that should be applicable to a wide range of domain states. Comparison with the truncated three mode amplitude equation description is made. (C) 1994 American Institute of Physics.
\end{abstract}

\section{INTRODUCTION}

The study of chaos in spatially extended systems where very many degrees of freedom are active in the dynamics ("spatiotemporal chaos") is proving a challenge to both experimentalists and theorists. There are a number of representative systems, experimental and numerical, showing chaotic dynamics and approaching to greater or lesser degree the ideal of large size with uniform external conditions. Examples, concentrating on two or higher dimensional systems, are the "spiral chaos" observed in experiments on RayleighBénard convection ${ }^{1}$ and numerical simulations of equations believed to approximately model such a system, ${ }^{2}$ electrohydronamic convection in nematic liquid crystals, 3,4 and numerical simulations of the complex Ginzburg-Landau equation. ${ }^{5,6}$ There is, however, little understanding of how to characterize such systems, for example to test in any systematic way the agreement between an experimental system and an attempt to model it theoretically, beyond the visual comparison of pictorial representations of the results. There is clearly a need for techniques to reduce the immense amount of data produced to allow meaningful comparisons and also simply to grasp the important physical processes occurring. In many cases regular spatial structures, that we may call coherent structures, are visually apparent in the complex dynamics (e.g. the spirals in the spiral chaos) and might provide appropriate collective coordinates for a reduced description, but so far such an approach has not been successfully pursued.

A surprising, but characteristic, feature of nonequilibrium systems is the formation of regular spatial structures above some critical driving strength. These structures often take the form of "stripe phases," such as the familiar parallel rolls of convecting fluid in the Rayleigh-Bénard system. As the system is taken further away from equilibrium a uniform stripe phase may break up into a complicated chaotic state of dynamic domains. A recent example is the breakdown of the stationary Turing pattern in a sustained chemical reaction system to a chaotic state. ${ }^{7}$ Alternatively, even if the parameters are such that a uniform stripe phase may be a stable steady state, the transient evolution towards this state from a random initial condition will typically pass through a coarsening domain state. This phenomenon has been studied experimentally in magnetic domains ${ }^{8}$ and ferrofluids, ${ }^{9}$ and in numerical simulations of the Swift-Hohenberg equation, ${ }^{10}$ although many interesting questions remain. Thus the ques- tion of the dynamics of disordered domain states is important in a diverse range of systems.

A particularly nice system for investigating these questions is provided by the system of a Rayleigh-Bénard cell rotated about a vertical axis. The study of this system was originally motivated as a simple system containing the basic physical ingredients of planetary atmospheric dynamics, namely buoyancy driven fluid flow perturbed by Coriolis forces. From the perspective of the questions outlined above, this system is of interest because it shows a novel instability, absent in the nonrotating case, known as the Kuppers-Lortz instability. ${ }^{11}$ This instability is manifested through a set of parallel rolls becoming unstable towards a second set of rolls rotated at a particular angle (at the onset of the instability) $\theta_{\mathrm{KL}}$. This second set of rolls, when established, in turn becomes unstable to a third set of rolls, rotated through a further $\theta_{\mathrm{KL}}$, and so on. No single set of rolls is a stable state, and neither is a superimposed roll state (forming a two dimensionally periodic or "lattice" structure) and a persistently dynamic state is expected. As we will see, in a Rayleigh-Bénard system of large aspect ratio (the ratio of radius in the horizontal plane to depth of the fluid, the appropriate measure of the "size" of the system) the dynamic state is in fact one of chaotic domains of variously oriented single-roll states. This system is particularly convenient to study, since the required experimental regime is quite attainable and also there are easily tuned control parameters that may be used to vary both the strength of the instability and the degree of nonlinearity (the rotation rate $\Omega$ and the Rayleigh number $R$, the dimensionless temperature difference across the cell). Also, from the theoretical perspective there is the advantage that the instability to the dynamic state occurs arbitrarily close to the threshold of the stripe state, so that the nonlinearity may be very weak, allowing a successful theoretical modeling through weakly nonlinear formulations such as the amplitude equation or other reduced equations.

Based on the fact that $\theta_{\mathrm{KI}}$ is very close to $\pi / 3$ for typical fluid parameter values, Busse and Heikes ${ }^{12}$ investigated a truncated model in which only three orientations of rolls were included, at angles of $\pi / 3$ with each other, with amplitudes $A_{i}, i=1 \ldots 3$. In addition they assumed spatially uniform states, leading to a dynamical model 


$$
\tau_{0} \frac{\partial A_{i}}{\partial t}=A_{i}\left(\epsilon-\sum_{j=1}^{3} g_{i j}\left|A_{j}\right|^{2}\right) \quad(i=1,2,3)
$$

where $\epsilon=\left(R-R_{c}\right) / R_{c}$ is the reduced Rayleigh number and $\tau_{0}$ sets the time scale. The magnitude of the amplitude can be scaled so that $g_{i i}=1$ for each $i$, and then by the rotational symmetry $g_{12}=g_{23}=g_{31}=g_{+}$and $g_{21}=g_{32}=g_{13}=g_{-}$, but, most importantly, $g_{+} \neq g_{\text {- because of the breaking of reflec- }}$ tion symmetry by the rotation. If we choose by convention $g_{+}>g_{-}$, then the single set of rolls $A_{1}=\epsilon^{1 / 2}$, becomes unstable towards the growth of $A_{2}$ for $g_{-} \leqslant 1$, no superimposed set is stable, and some sort of persistent dynamics is expected. In fact the dynamics of Eq. (1) is controlled by the heteroclinic orbit connecting the three unstable fixed points: $\left(\epsilon^{1 / 2}, 0,0\right),\left(0, \epsilon^{1 / 2}, 0\right)$ and $\left(0,0, \epsilon^{1 / 2}\right)$ for the triad $\left(A_{1}, A_{2}, A_{3}\right)$. Numerical evolution of the equations shows that the orbit steadily approaches the heteroclinic orbit, spending longer and longer in the vicinity of the three unstable fixed points, before rapidly switching to the vicinity of the next fixed point. Busse and Heikes suggested that eventually the slowing down of the orbit would be quenched by inevitable extrinsic noise in an experimental system. This would lead to the interesting result of a macroscopic fluid system with dynamics (e.g. the switching time) controlled by microscopic noise sources, perhaps thermal noise. They called such a state "statistical chaos."

From the earliest experiments, ${ }^{13}$ as well as more recent ones, ${ }^{14}$ it is clear that for experimental systems that are large, where lateral boundary effects may not play a significant role in nucleating successive roll transitions, the state is by no means spatially homogeneous, but is instead one of fluctuating domains. Tu and Cross ${ }^{15}$ suggested that the effective interaction between the different domains would take over the role of the extrinsic noise in the Busse-Heikes dynamics, leading to an intrinsically chaotic state. They investigated this idea by simply adding the standard "NewellWhitehead-Segel" spatial derivative terms ${ }^{16}$ to Eq. (1) and numerically studied the spatially inhomogeneous solutions of the equations

$$
\tau_{0} \frac{\partial A_{i}}{\partial t}=A_{i}\left(\epsilon-\sum_{j=1}^{3} g_{i j}\left|A_{j}\right|^{2}\right)+\xi_{0}^{2}\left(\hat{q}_{i} \cdot \nabla\right)^{2} A_{i} \quad(i=1,2,3)
$$

where $\hat{q}_{i}, i=1 \ldots 3$ are the three directions of the sets of rolls at angles $i \pi / 3$. Notice that for a boundary between two domains of rolls that is not orthogonal to either set of rolls (so that $\hat{q}_{i}, \nabla A_{i} \neq 0$ ) the length scale of the domain wall is $O\left(\epsilon^{-1 / 2}\right)$, and the usual transverse derivative terms, involving $i\left(\hat{q}_{i} \times \nabla\right)^{4} A_{i}$, will be negligible. This led Tu and Cross to ignore such terms which are more difficult to handle numerically and force the consideration of complex equations, including however a term in $\left(\hat{q}_{i} \times \nabla\right)^{2} A_{i}$ with small coefficient for numerical stability. They then simulated Eq. (2) for real $A_{i}$. The results did indeed show intrinsically chaotic domain states, qualitatively similar to the experiment.

The Tu-Cross model has a number of imperfections as an accurate representation of the fluid system. It follows Busse and Heikes ${ }^{12}$ in truncating the continuous range of possible orientations to a discrete set of three. (Note that because real amplitudes were taken, not even small reorientations of the rolls, which may be captured by a spatially dependent phase of a complex amplitude, were allowed.) This might be expected to be particularly important well into the unstable regime, where the instability is to a wide range of possible angles around $\theta_{\mathrm{KL}}$. Also no distribution in the wave vector of the rolls is allowed, and the only defect in the regular stripe phase expected to occur in the model are the "amplitude domain walls" between two domains where the amplitude of one set of rolls decreases as the amplitude of the second set grows: neither "phase domain walls" where the orientation of the rolls rapidly changes, or dislocations are included.

In this paper we investigate a more general model of the rotating domain state that overcomes, at the cost of increased numerical difficulty, these objections. The model we use is based on the Swift-Hohenberg equation, ${ }^{17}$ which has successfully been used to model a wide range of phenomena involving the steady states, the relaxation towards these states, and the effect of extrinsic noise, on nonrotating convection and other pattern formation systems showing steady stripe phases. We modify this equation in the simplest way to incorporate the Kuppers-Lortz instability of the rotating system. It is interesting to point out that adding the simplest term that breaks the chiral symmetry, as the rotation does in the physical system, converts a purely relaxational system (known as a gradient or potential system) into one with no potential for which persistent dynamics is possible. As we will see, persistent dynamics does indeed occur.

One goal of our investigation of this model is to test the validity of the Tu-Cross amplitude equations and the predictions of this approach. The new model allows for the full range of possible roll orientations: we would like to know whether the chaotic dynamics of the amplitude equation survives this generalization, and whether its qualitative characteristics change. For example, Tu and Cross found that the chaotic motion survives as a persistent state into the range of parameters for which a uniform stripe phase is also stable, i.e. the system is bistable in this parameter range. In addition we can test the quantitative aspects of the predictions of the amplitude equation, for example the predicted scaling of the domain size with $\epsilon^{-1 / 2}$, and the dependence of the characteristic length and time scales, through the nonlinear coefficients, on the rotation rate.

A second goal is to investigate the chaotic domain state in the model as a representative example of spatially extended chaos. Given a chaotic state, be it from numerical simulation or experiment, that to the eye appears to be one of domains with dynamic domain walls or grain boundaries, can one develop methods to analyze the data to qualitatively display this idea, and then to quantitatively characterize the structure? The chaotic domain state is particularly appropriate to test such methods since the coherent structures that are evident to visual observation turn out, as we will see, to be quite easy to extract numerically. Other cases, such as the spiral structures in nonrotating convection, may provide a less good match to pre-existing tools.

A final application of the new model, which is left to 
future work, is to investigate the effects of finite geometries-inevitably a complication of real experimental systems-for example to see how the chaotic dynamics is suppressed as the characteristic scale of the chaotic state approaches the system size. In the case of rotating convection it is also of interest to understand the interaction of the chaotic domain state in the bulk with the wall localized traveling wave state. ${ }^{18}$

\section{THE MODEL}

The model we simulate is given by the equation of motion of a real field $\psi$ which is a function of the two horizontal space coordinates $x, y$ :

$$
\begin{aligned}
\partial_{t} \psi= & \epsilon \psi-\left(\nabla^{2}+1\right)^{2} \psi-g_{1} \psi^{3}+g_{2} \hat{z} \cdot \nabla \times\left[(\nabla \psi)^{2} \nabla \psi\right] \\
& +g_{3} \nabla \cdot\left[(\nabla \psi)^{2} \nabla \psi\right] .
\end{aligned}
$$

Retaining only the first three terms on the right-hand state leads to the familiar Swift-Hohenberg equation. The equation then shows an instability when the control parameter $\epsilon$ passes through zero to a state with spatial structure varying as $\cos [\hat{q} \cdot \mathbf{x}]$ corresponding to stripes with normals in the $\hat{q}$ direction. The length scale in Eq. (3) has been chosen so that the wave number of the stripe pattern at onset is unity. As a model of convection, for example, the parameter $\epsilon$ is the reduced Rayleigh number $\epsilon=\left(R-R_{c}\right) / R_{c}$ with $R$ the Rayleigh number and $R_{c}$ its critical value giving the first onset of convection. The field $\psi$ is then a representation of the structure of this state in the horizontal plane, for example it could represent the temperature at the midplane of the convection cell. (For a discussion of the use of this equation as a rotationally invariant version of the amplitude equation and as a useful model outside of any controlled perturbative derivation, see Ref. 16.)

The fourth term on the right-hand side of Eq. (3), $g_{2} \hat{z} \cdot \nabla \times\left[(\nabla \psi)^{2} \nabla \psi\right]$, is the all important term that breaks the reflection or chiral symmetry, and represents the effect of rotation on the convection system. It is the simplest term constructed out of $\psi$ and its derivatives retaining the inversion symmetry $\psi \rightarrow-\psi$, the parity symmetry $\mathbf{x} \rightarrow-\mathbf{x}$, and the rotational invariance in the $x, y$ plane. In a derivation of Eq. (3) from the fluid equations we would expect $g_{2}$ to increase with the rotation rate $\Omega$, and be proportional to $\Omega$ for small rotations.

The reason for including the final term $g_{3} \nabla \cdot\left[(\nabla \psi)^{2} \nabla \psi\right]$ is shown by calculating the amplitude equation corresponding to Eq. (2) resulting from this model. Writing

$$
\psi=\sum_{j} A_{j} \exp \left[i \hat{q}_{j} \cdot \mathbf{x}\right]
$$

leads to an amplitude equation as in Eq. (2) with $\xi_{0}=2$, $\tau_{0}=1$, and $g_{i j}=g\left(\theta_{i j}\right)$ with $\theta_{i j}$ the angle between the rolls $\cos \theta_{i j}=\hat{q}_{i} \cdot \hat{q}_{j}$, and

$$
\begin{aligned}
& g(\theta)=6 g_{1}+2 g_{2} \sin 2 \theta+2 g_{3}(2+\cos 2 \theta), \quad \theta \neq 0, \\
& g(0)=\frac{1}{2} g(\theta \rightarrow 0)=3 g_{1}+3 g_{3} .
\end{aligned}
$$
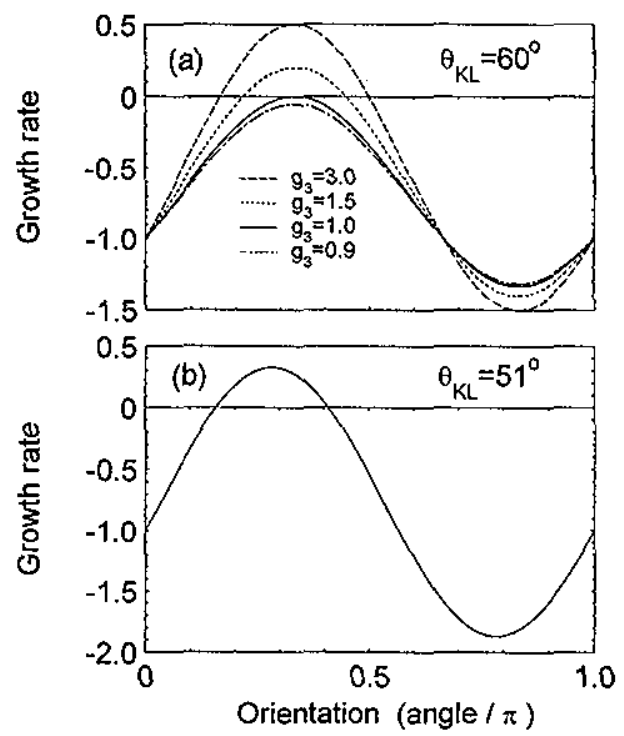

FIG. 1. Growth rate of the Kuppers-Lortz instability as a function of the orientation of the new set of rolls for Eq. (3): (a) Maximum growth rate at $60^{\circ}\left(g_{2}=-\sqrt{3} g_{3}\right)$ for various values of $g_{3} ;$ (b) Maximum growth rate at $51^{\circ}\left(g_{2}=-4.70 g_{3}\right)$ for $g_{3}=0.522$.

The Kuppers-Lortz instability, when stripes $i$ become unstable towards stripes $j$, first occurs for the angle $\theta_{i j}=\theta_{\mathrm{KL}}$ that gives $g\left(\theta_{i j}\right)$ its largest negative value. Minimizing $g(\theta)$ yields

$$
\tan 2 \theta_{\mathrm{KL}}=g_{2} / g_{3},
$$

and the instability then occurs for

$$
3 g_{1}+g_{3}-2 \sqrt{g_{2}^{2}+g_{3}^{2}}<0 .
$$

Thus we may use the coefficient $g_{3}$ to tune $\theta_{\mathrm{KL}}$.

We may choose, for example, $\theta_{\mathrm{KL}}$ to be close to $\pi / 3$ as in the experimental system. This value occurs for $g_{2}=-\sqrt{3} g_{3}$ and the instability then occurs for $g_{3}<g_{1}$. Note that by rescaling the magnitude of $\psi$ we may choose $g_{1}$ to be of unit magnitude, and in the remainder of the paper we will always make this choice. The Kuppers-Lortz instability at an angle $\theta_{\mathrm{KL}}=\pi / 3$ then occurs at $g_{1}=1$, $g_{2}=-\sqrt{3}, g_{3}=1$. More generally, still fixing $g_{1}=1$, $g_{2}=-\sqrt{3} g_{3}$ so that the maximum growth rate occurs at $\pi / 3$, we can scan through the instability by varying $g_{2}, g_{3}$ to give Eq. (2) with $g_{+}=2, g_{-}=2 /\left(1+g_{3}\right)$. The growth rate of the instability as a function of the angle of the second set of rolls for various values of $g_{3}$ is shown in Fig. 1(a). Notice that for $g_{3}>1$, although the maximum growth rate occurs at the angle $\pi / 3$, there is a band of unstable angles, so that the domain angle of the new set of rolls is not necessarily fixed at $\pi / 3$. This effect is not included in the three mode truncation of Busse and Heikes or Tu and Cross, but does occur in the fluid system and may be studied using our more general equation, Eq. (3). Also for $g_{3}<1$, where Tu and Cross found bistability in the three mode truncation, it is much less clear that the truncation is reliable: the angle $\pi / 3$ is only singled out as the least rapidly decaying perturbation in the linear stability analysis. 


\section{NUMERICAL METHOD}

As the spatial domain associated with Eq. (3) is periodic in both the $x$ and $y$-directions, a spectral technique is an obvious choice for numerical integration of this nonlinear PDE (cf. for example Canuto et al. ${ }^{19}$ ). In this approach we apply the discrete Fourier transform on the solution $\psi(x, y, t)$ in order to generate a numerical approximation for the finite Fourier transform of $\psi, \bar{\psi}\left(q_{x}, q_{y}, t\right)$, defined by

$\psi(x, y, t)=\sum_{q_{x}=-\infty}^{\infty} \sum_{q_{y}=-\infty}^{\infty} \tilde{\psi}\left(q_{x}, q_{y}, t\right) \exp \left(i q_{x} x\right) \exp \left(i q_{y} y\right)$.

Since the field $\psi$ is real, the modal amplitudes with negative wave number are related to their counterparts with positive wave number through complex conjugation:

$$
\tilde{\psi}\left(q_{x}, q_{y}, t\right)=\overline{\bar{\psi}\left(-q_{x},-q_{y}, t\right)} .
$$

Using this representation for $\psi$ it is straightforward to compute the various spatial derivatives required to assemble the nonlinear term. The modal amplitudes $\tilde{\psi}\left(q_{x}, q_{y}, t\right)$ are related to the field $\psi(x, y, t)$ through the finite Fourier transform:

$$
\begin{aligned}
\tilde{\psi}\left(q_{x}, q_{y}, t\right)= & \frac{1}{L_{x} L_{y}} \int_{0}^{L_{x}} d x \int_{0}^{L_{y}} d y \quad \psi(x, y, t) \\
& \times \exp \left(-i q_{x} x\right) \exp \left(-i q_{y} y\right) .
\end{aligned}
$$

In order to approximate the amplitudes $\tilde{\psi}$ we sample the field $\psi$ on the regular grid

$$
\begin{aligned}
& x_{i}=\frac{(i-1) L_{x}}{N_{x}}, \quad i=1, \ldots, N_{x}, \\
& y_{j}=\frac{(j-1) L_{y}}{N_{y}}, \quad j=1, \ldots, N_{y}
\end{aligned}
$$

with $L_{x}$ and $L_{y}$ the dimensions of the region in the $x$ and $y$ directions, and apply the trapezoidal rule to Eq. (10):

$\tilde{\psi} \approx \frac{1}{N_{x} N_{y}} \sum_{i=1}^{N_{x}} \sum_{j=1}^{N_{y}} \psi\left(x_{i}, y_{j}, t\right) \exp \left(-i q_{x} x_{i}\right) \exp \left(-i q_{y} y_{j}\right)$.

The wave numbers are restricted to the set $\left(q_{x}\right)_{i}=2 \pi i / L_{x}$ and $\left(q_{y}\right)_{j}=2 \pi j / L_{y}$ with

$$
-\left(N_{x} / 2-1\right) \leqslant i \leqslant N_{x} / 2, \quad-\left(N_{y} / 2-1\right) \leqslant j \leqslant N_{y} / 2 .
$$

For linear equations, methods based on such discrete transforms provide optimal accuracy in that the rate of convergence for suitably smooth solutions is faster than any power of $N_{x}$ and $N_{y} .{ }^{19}$ In addition this technique avoids the phase errors commonly associated with finite difference methods. Equation (3) is of course nonlinear. Thus for a finite collection of amplitudes construction of the nonlinear term will lead to aliasing of the modes. For cubic nonlinearity this results in contamination of one-half of the available modes. Such aliasing contributions can lead to unphysical instabilities. While our numerical implementation has the capability to "dealias" the affected modes the strong damping provided by the linear biharmonic term appears to render unnecessary the need for this provided there is sufficient resolution of the solution. This is tested by reviewing the intensity of the large wave number Fourier modes, and by testing the consistency of the quantitative results (such as the correlation lengths plotted in Fig. 8 below) against doubling the mesh resolution. Given the solution at time $t$ we advance the solution numerically to the next time level $t+\Delta t$ by the following procedure. Since the linear terms involve a biharmonic operator, the implementation of an explicit time-marching procedure would require the use of an unacceptably small time step due to the stiffness associated with such an operator. In order to avoid this, all linear terms are integrated using an exponential propagation procedure described in detail below with the nonlinear terms being handled explicitly. While it is in principle possible to construct a fully implicit procedure using the ideas espoused by Friesner $e t$ al ${ }^{20}$ this has not been pursued here. For the purposes of time integration we write Eq. (3) in the form

$$
\partial_{t} \psi=\epsilon \psi-\left(\nabla^{2}+1\right)^{2} \psi+N(\psi, t),
$$

where $N(\psi, t)$ denotes the nonlinear term given by

$$
\begin{aligned}
N(\psi, t)= & -g_{1} \psi^{3}+g_{2} \hat{z} \cdot \nabla \times\left[(\nabla \psi)^{2} \nabla \psi\right] \\
& +g_{3} \nabla \cdot\left[(\nabla \psi)^{2} \nabla \psi\right] .
\end{aligned}
$$

Taking the Fourier transform of both sides we obtain

$$
\partial_{t} \tilde{\psi}=\alpha\left(\epsilon, q_{x}, q_{y}\right) \tilde{\psi}+\bar{N}(\tilde{\psi}, t),
$$

where

$$
\alpha=\epsilon-\left[1-\left(q_{x}^{2}+q_{y}^{2}\right)\right]^{2} .
$$

In our numerical implementation, the transformed nonlinear term $\tilde{N}$ is evaluated using the pseudospectral method in which derivatives are evaluated in Fourier space while products are evaluated in physical space. As mentioned above, this has the disadvantage that fully one-half of the modes are subject to aliasing. Equation (16) can be treated as a linear system of differential equations modified by a nonlinear forcing term. The nonlinear terms are integrated by a predictorcorrector method. ${ }^{21}$ Multiplying both sides by an appropriate integrating factor we obtain

$$
\left.\exp (-\alpha t) \tilde{\psi}\right|_{t} ^{t+\Delta t}=\int_{t}^{t+\Delta t} d t^{\prime} \tilde{N}\left(\tilde{\psi}, t^{\prime}\right) \exp \left(-\alpha t^{\prime}\right) .
$$

We next assume that the nonlinear term $\tilde{N}$ can be approximated over the interval $t \leqslant t^{\prime} \leqslant t+\Delta t$ by a linear function of 't':

$$
\tilde{N} \approx \tilde{N}_{0}+\tilde{N}_{1}\left(t-t^{\prime}\right),
$$

where

$$
\begin{aligned}
& \tilde{N}_{0}=\tilde{N}(\psi(t), t), \\
& \tilde{N}_{1}=\frac{\tilde{N}(\psi(t+\Delta t), t+\Delta t)-\tilde{N}(\psi(t), t)}{\Delta t} .
\end{aligned}
$$

Note that we are proposing to integrate the nonlinear term explicitly through this procedure. Thus our algorithm will be subject to a stability restriction on the time step $\Delta t$. In principle, this can also be avoided through the use of a 
fully nonlinear implicit technique but we have not pursued this since empirically the time step restriction imposed by the nonlinear term is not observed to be particularly onerous. The final algorithm for time marching then has the form

$$
\begin{aligned}
\tilde{\psi}(t+\Delta t)= & \exp (\alpha \Delta t) \tilde{\psi}(t)+\tilde{N}_{0}\left[\frac{\exp (\alpha \Delta t)-1}{\alpha}\right] \\
& +\tilde{N}_{1}\left[\frac{\exp (\alpha \Delta t)-(1+\alpha \Delta t)}{\alpha^{2}}\right]
\end{aligned}
$$

In order to evaluate $\tilde{N}_{1}$ we use as an approximate value of $\tilde{\psi}\left(q_{x}, q_{y}, t+\Delta t\right)$ the value obtained by applying Eq. (20) but with $\tilde{N}_{1}=0$ and then reevaluate Eq. (20) with this guess. This "predictor-corrector" step can be repeated until convergence is obtained but in practice we perform this step only once. It can be shown that the overall technique is secondorder accurate in $\Delta t$ and this has been verified in our numerical tests of this procedure. Finally, we note that our entire procedure is highly vectorizable as well as parallelizable. A typical application of the algorithm for the type of problems considered here employed a resolution of $N_{x}=512$ and $N_{y}=512$ with a typical time step of $\Delta t=0.1$ or 0.2 . One time step at this resolution required $0.8 \mathrm{~s}$ on a Cray Y/MP.

\section{RESULTS}

\section{A. Spatial pattern}

An example of an instantaneous pattern is shown in Fig. 2(a). The parameters for this figure are $\epsilon=0.3, g_{3}=1.5$, (with $g_{1}=1, g_{2}=-\sqrt{3} g_{3}$ ). The system size is $80 \pi$, so that the square side would contain 40 stripe periods, and the time is 1600 after a random initial condition. It is immediately apparent that the predominant structure is one of domains of differently oriented rolls, with rather sharp grain boundaries separating them, together with a few more complicated regions that appear to be superimposed roll structures. The dynamics of the state, shown by comparing many such plots and also by plotting $\partial_{t} \psi$, is dominated by the motion of the grain boundaries so that one domain consumes its neighbor in turn to be consumed by its neighbor-the orientation of the rolls within a domain does not otherwise evolve. The Fourier intensity of this pattern $S_{1}(q)$ is shown in Fig. 2(b). [We denote this by $S_{1}$, reserving $S(q)$ for the time averaged structure factor.] There is a strong peaking of the intensity around the circle $|\mathbf{q}|=1$, the critical wave vector for Eq. (2). In addition an interesting structure is apparent in the angular structure. Since the radial structure of the Fourier intensity is what is expected from the structure of the equation-the $\left(\nabla^{2}+1\right)^{2}$ operator provides strong filtering away from $q=1-$ it is more useful to concentrate on the angular structure. We therefore integrate the Fourier intensity over radial coordinates to give the angular intensity as a function of the direction (given by the unit vector $\hat{q}$ with $\mathbf{q}=q \hat{q}$ )

$$
\bar{S}_{1}(\hat{q})=\int 2 \pi q d q S_{1}(q, \hat{q}) .
$$

We may now plot this quantity as a function of time to give an overview of the evolution of the domain structure. This is shown in Fig. 3.

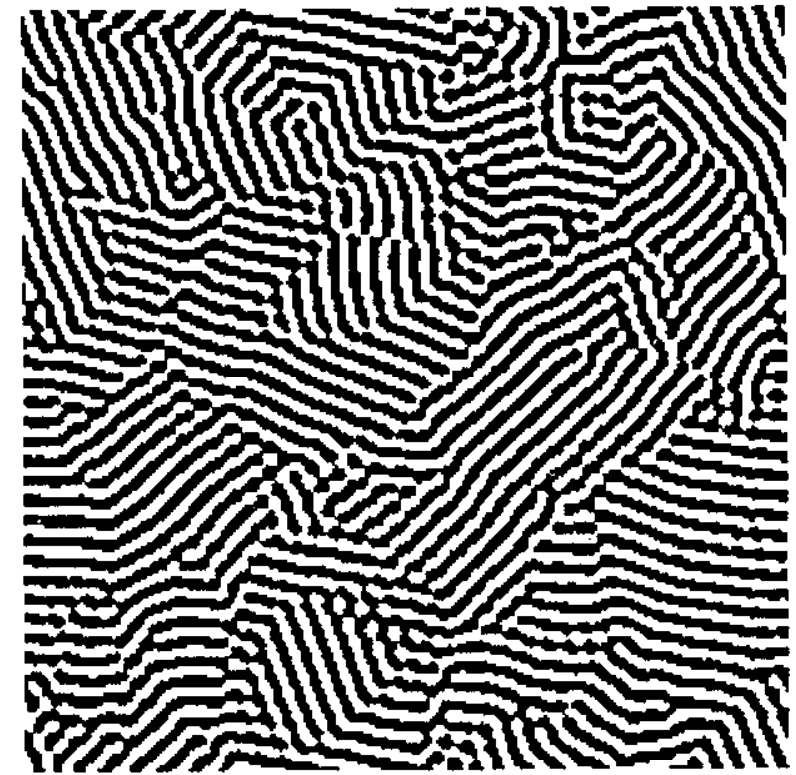

(a)

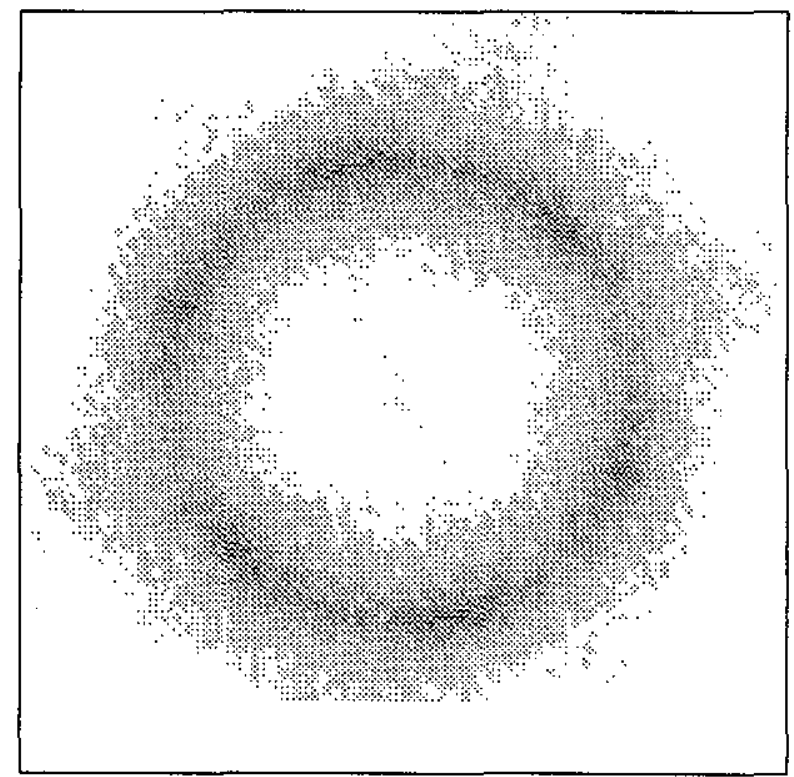

(b)

FIG. 2. Field $\psi(\mathbf{r})$ (top panel) and Fourier transform $\tilde{\psi}(\mathbf{q})$ (bottom panel) after a time 1600 from random initial conditions showing a snapshot of the characteristic domain structurc. The parameters are $g_{1}=1, g_{2}=-2.60$, $g_{3}=1.5$ and $\epsilon=0.3$, in a system of side $80 \pi$. In the field representation (top panel) black denotes positive values and white negative values. In the bottom panel the full range of wave vectors shown is \pm 1.6 in each direction $q_{x}, q_{y}$.

Although all possible domain angles are allowed in the pattern, the remarkable switching phenomena shown in the three mode truncation is immediately apparent in these results. Indeed the plot of $S_{1}(q)$ at some time shows that the intensity is quite strongly peaked at six orientations spaced approximately uniformly around the circle. If we interpret $S_{1}(\hat{\mathbf{q}})$ approximately as the fraction of the system occupied 


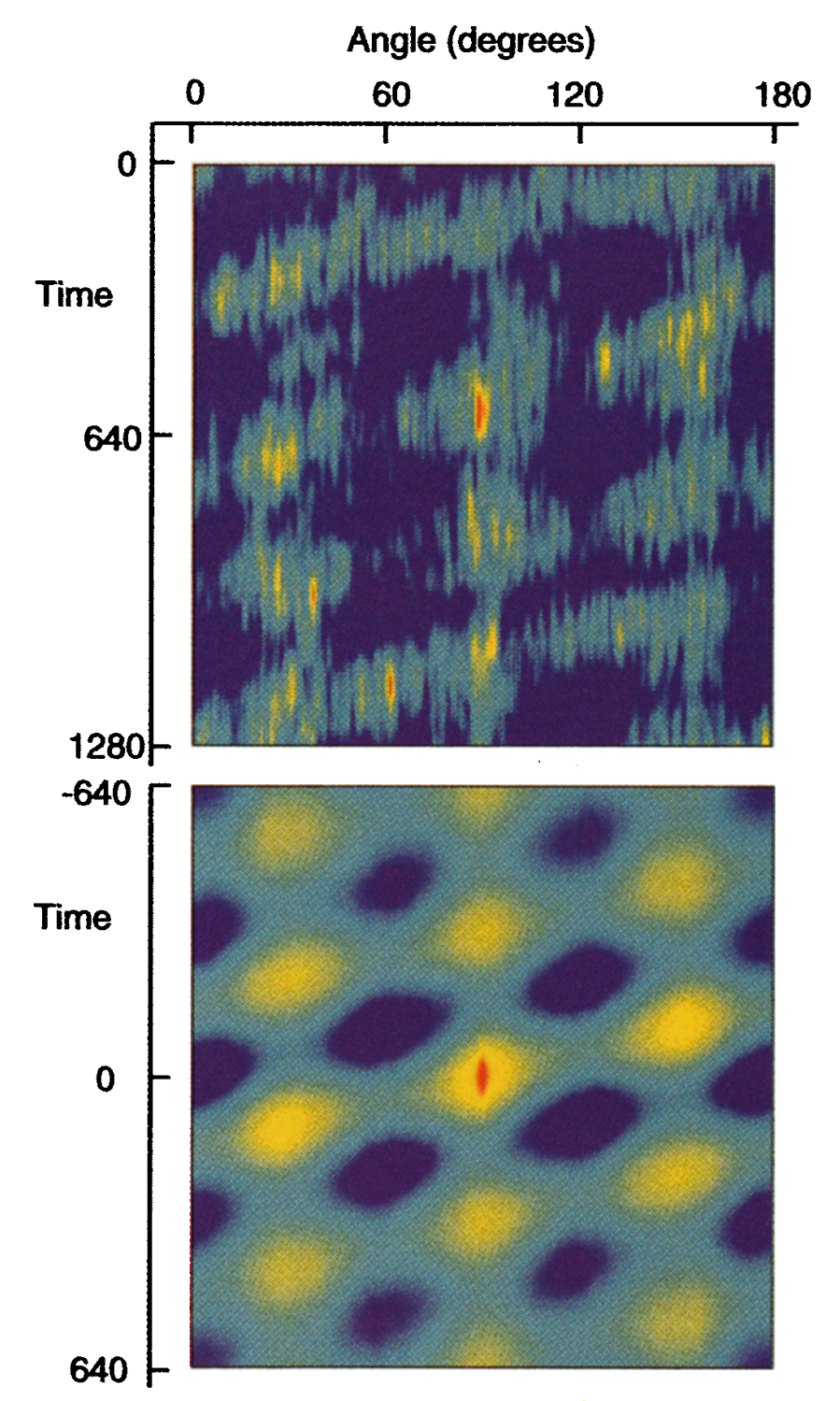

FIG. 3. Angular Fourier intensity versus time plot and its correlation function. Top panel is a color scale plot of the radially integrated Fourier intensity (blue, zero; red maximum intensity) as a function of the angle $\theta_{q}$ of the wave vector, plotted against time for an interval of length 1280 starting at time 320 for the same parameters as in Fig. 2 but with a system size of $120 \pi$. The lower panel is the correlation function of the top panel treated as a two-dimensional image, with the origin $\left(\theta_{q}=0, t=0\right)$ in the center of the plot.

by domains of orientation $\hat{q}$, we see that a three orientation approximation might indeed be a good representation. The switching behavior can be clearly displayed by plotting the correlation function of the space-time structure shown in Fig. 3(a). This is shown in Fig. 3(b) with $t=0, \theta_{q}=0$ at the center of the plot. The strong peak at time $t \simeq 100$, yields the characteristic switching time $\tau_{s} \simeq 100$ to domains at an angle $\theta_{s}$ very close to $-\pi / 3$. The correlation remains peaked at integral multiples of this $\left(\theta_{s}, \tau_{s}\right)$ offset, decaying slowly with increasing time showing the effect of the chaotic components of the dynamics which are apparent in the plot of $S_{1}(\hat{\mathbf{q}}, t)$ [Fig. 3(a)]. Figures 3(a) and 3(b) should be compared with the similar plots in Zhong and Ecke $^{22}$ (see also Ning and $\mathrm{Ecke}^{23}$ ), which inspired this approach: the similar- ity is indeed striking. It is worth pointing out that the estimate of the time $\tau_{s}$ involves rather a complicated procedure. First, the nonuniform distribution of Fourier intensity with angle, since it involves an average over the whole pattern, is a finite size effect (or a reflection of long range orientational order in the pattern). The switching seen in Figs. 3(a) and $3(b)$ is in the orientation of domains that dominate this Fourier average-not necessarily the switching of a single domain. In addition, even if the observed switching is largely determined by the switching of single domains, it is clearly the largest domains that will dominate the average, and so $\tau_{s}$ is a reflection of the properties of these domains, rather than an average with some known weight over the switching times of all the domains. Nevertheless we will see that the time identified in this way seems consistent with other definitions discussed below.

An interesting question that we have not been able to fully answer is the question of the possible long range orientational order in these structures. The concentration of domain intensity at a discrete set of orientations may be a finite size effect that would go away as the system size increases when very distant regions of the system are effectively independent. In this case the three mode truncation would be a good local approximation, but not a global characterization of the whole pattern. On the other hand, the effect could persist for arbitrarily large system sizes, in which case the chaotic domain state would truly show long range orientational order, whilst still being disordered, through the existence of domains, in the translational degrees of freedom. We will return to this question when we discuss more quantitatively the spatial structure, although because of the limitation of the system sizes that are practical to simulate and possible delicate dependence on the underlying numerical mesh, we do not have a definitive answer to this question.

\section{B. Domain structure}

To do a systematic characterization of the patterns, particularly statistical averages over a long time evolution rather than a description of individual representatives, we need a technique to extract the domain structure. A similar problem has arisen in other systems: ${ }^{7,8}$ these authors have successfully extracted the domain structure for individual patterns, but have not attempted a statistical description. We have found that a modification of the method of Ref. 7, essentially following a proposal of Ref. 24, works remarkably well. This technique has been called a wavelet transform, but can be simply understood as a Fourier space filtering technique identical to focal plane optical filtering. Taking advantage of the collapse of the Fourier intensity onto the $q=1$ ring makes this approach particularly easy to implement in the present case.

We first define a filtered Fourier transform of an individual pattern as the product of the Fourier transform $\tilde{\psi}(\mathbf{q})$ of the field $\psi(\mathbf{r})$ with a Gaussian filter centered at some angle $\theta_{f}$ around the $q=1$ circle in Fourier space:

$$
\tilde{\psi}_{f}\left(\mathbf{q}, \theta_{f}\right)=e^{-\left(\mathbf{q}-\mathbf{q}_{f}\right)^{2} / \sigma^{2}} \tilde{\psi}(\mathbf{q})
$$


where $\mathbf{q}_{f}=\left(\cos \theta_{f}, \sin \theta_{f}\right)$ and $\sigma$ sets the width of the filtering function. If we perform the inverse Fourier transform with respect to $\mathbf{q}$ on $\psi_{f}\left(\mathbf{q}, \theta_{f}\right)$ we get a function $\psi_{f}\left(\mathbf{r}, \theta_{f}\right)$ that is large in magnitude in those domains where the stripe normal is close to the $\theta_{f}$ direction. (This is the same procedure used by Ref. 7 who phrased the procedure as a convolution in real space.) This picks out domains of a chosen orientation, but does not directly give the domain angle as a function of position. We modify this method by considering now the dependence of $\psi_{f}$ on $\theta_{f}$ : we calculate $\psi_{f}\left(\mathbf{r}, \theta_{f}\right)$ as a function of $\theta_{f}$ and define the orientation $\theta_{d}(\mathbf{r})$ of the stripes at each point $\mathbf{r}$ as the value of $\theta_{f}$ that maximizes the magnitude $\left|\psi_{f}\left(\mathbf{r}, \theta_{f}\right)\right|$ of the filtered field. Since the filtering and inverse Fourier transform are time and memory intensive numerical operations, in practice we choose a discrete set of $\theta_{f}$ and fit a parabola to sets of three neighboring points to determine the maximum. Typically we have used 8 values around the semicircle at $q=1$ [since $\tilde{\psi}(-\mathbf{q})=\overline{\tilde{\psi}(\mathbf{q})}$ for the real field $\psi$ the other half of the circle does not give independent information] with $\sigma=0.5$, but find no apparent change in the resulting structure using for example 12 angles. [For an ideal state of parallel stripes we expect an error of about $\pm 1.5^{\circ}$ in the angle $\theta_{d}$ determined using 8 windows of width 0.5 , reducing to $0.5^{\circ}$ for 12 windows. However because of the discrete nature of the crude maximization procedure used, the derivative $d \theta_{d} / d \theta$ deviates significantly from unity-by as much as $25 \%$ for 8 windows and $10 \%$ for 12 windows-with the deviation showing rather sharp spikes at the window borders. This introduces some spurious structure at this level into a histogram of the distribution of orientations, which involves such a derivative. Note that since the maximization procedure is site-local, whereas the function to be maximized is produced by a global calculation (fast Fourier transforms), iterative methods to home in to the maximum will be very inefficient. If more accurate histograms are desired, a higher order interpolation scheme seems to be the best solution, or the effect of the filtering on a uniform distribution can be factored out of the results.] As a by-product of the domain filtering algorithm we can also directly pick out the domain walls and defected regions (e.g. the regions of stripe superposition) as regions where the maximum magnitude $\left|\psi_{f}\left(\mathbf{r}, \theta_{d}\right)\right|$ is reduced.

To illustrate these procedures we show in Fig. 4 an example of the field $\psi(\mathbf{r})$ for a particular time, together with the reconstructed domain pattern as well as the domain wall pattern. It can be seen that the algorithm quite faithfully captures the domain structure, and we can now study the patterns and dynamics within this simpler reduced description. The final panel in Fig. 4 shows a histogram of the distribution of stripe orientations. The strong concentration into peaks separated in angle by $\pi / 3$ is quite striking. It is interesting to compare Fig. 4 with the results of the three mode amplitude equation model [Fig. 1(a) of Ref. 15]: there is a strong resemblance, even though, it should be emphasized, the present results are calculated and plotted with the full continuum of possible orientations.

Figure 5(a) shows the time trace for the stripe orientation $\theta_{d}$ as a function of time at a particular point in the pattern. Again the domain switching by angles close to $\pi / 3$ are evi- dent, as is also the fact that there are distributions both of the switching angle and the switching time. As might be expected from the breadth of the latter distribution the power spectrum of the time trace shows broad structures and broadband noise. The power spectrum for eight different traces at points throughout the region, and their average, are shown in Fig. 5(b). We can identify a characteristic time from the peak at around $\omega=0.02$ to give a time $\tau_{d} \simeq 300$. Since a complete period corresponds to three switches through about $\pi / 3$, this time is consistent with the single switch time $\tau_{s} \approx 100$ identified from the Fourier distribution versus time plot.

\section{Quantitative analysis and comparison with amplitude equation model}

We would now like to characterize the domain structures more quantitatively, to compare with the predictions of the three mode truncated model, and also to provide a basis for comparison with experiment.

A natural first step is to define a correlation length that captures the length scale of the pattern, or naively the domain size. We have done this using the correlation function of the filtered field $\psi_{f}(\mathbf{r})$. The correlation function of the filtered field for a particular filtering angle $\theta_{f}$ is defined as

$$
\begin{aligned}
C\left(\mathbf{R} ; \theta_{f}\right)= & <\psi_{f}\left(\mathbf{r}, t ; \theta_{f}\right) \psi_{f}\left(\mathbf{r}+\mathbf{R}, t ; \theta_{f}\right)>_{\mathbf{r}, t} \\
& -<\psi_{f}\left(\mathbf{r}, t ; \theta_{f}\right)>_{\mathbf{r}, t}^{2}
\end{aligned}
$$

and we define the filtered correlation function as this quantity averaged over $\theta_{f}$

$$
C_{f}(\mathbf{R})=\frac{1}{n_{f}} \sum_{f=1}^{n_{f}} C\left(\mathbf{R}, \theta_{f}\right)
$$

summing over the $n_{f}$ discrete filtering angles. This function is plotted for separations $\mathbf{R}$ along the $\mathbf{x}$ and $\mathbf{y}$ directions for the same parameters as Figs. 2 and 3 in Fig. 6. (Note that the integral of $C$ over all $\mathbf{R}$ is zero, so that there is a small apparent anticorrelation at large separations.) Although an exponential is an approximate fit to the decay of this function, we have found that an easier way to extract a characteristic length, that is also less sensitive to changing the system size, is simply to take the width at half the maximum of $C_{f}$ : we will call the correlation length calculated in this way $\xi_{f}$. The variation of this correlation length with the important parameters is shown in Fig. 7.

For comparison with the amplitude equation we show the variation with the control parameter $\epsilon$ in $(a)$. In the amplitude equation description of Tu and Cross $\epsilon$ may be eliminated from the equation by defining a scaled length variable $\mathbf{X}=\epsilon^{1 / 2} \mathbf{x} / \xi_{0}$. Thus according to this description the correlation length should scale with $\epsilon^{-1 / 2}$ at least for small $\epsilon$ with perhaps higher order corrections coming in for larger $\epsilon$. It is hard to use large enough systems sizes and correspondingly long equilibration times to unambiguously confirm this asymptotic scaling for small $\epsilon$. We can see, however, that the inverse correlation length measured from the numerical simulations and shown in Fig. 7(a) clearly decreases as $\epsilon$ decreases, and is well fit by an expression of the form 


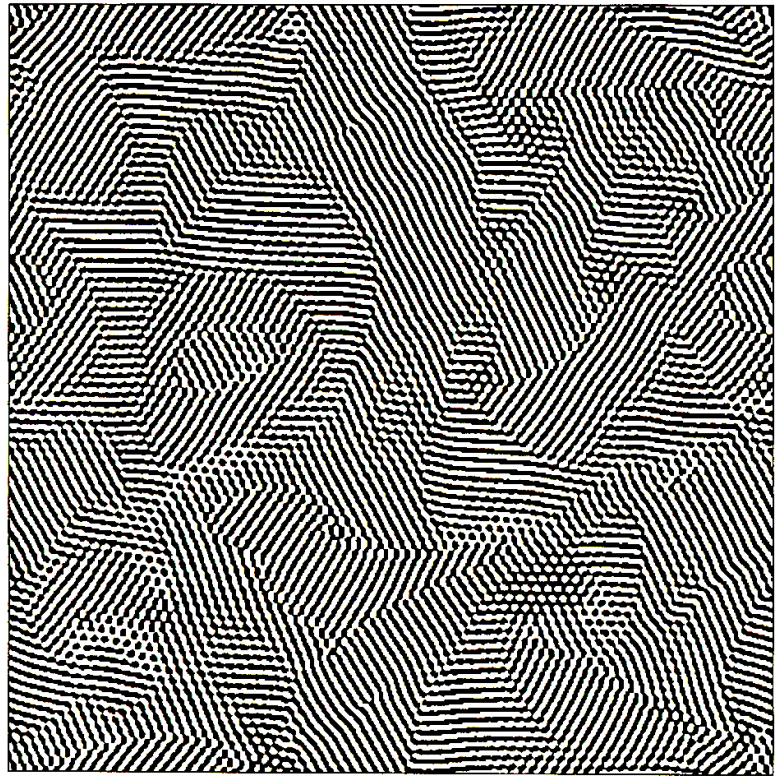

(a)

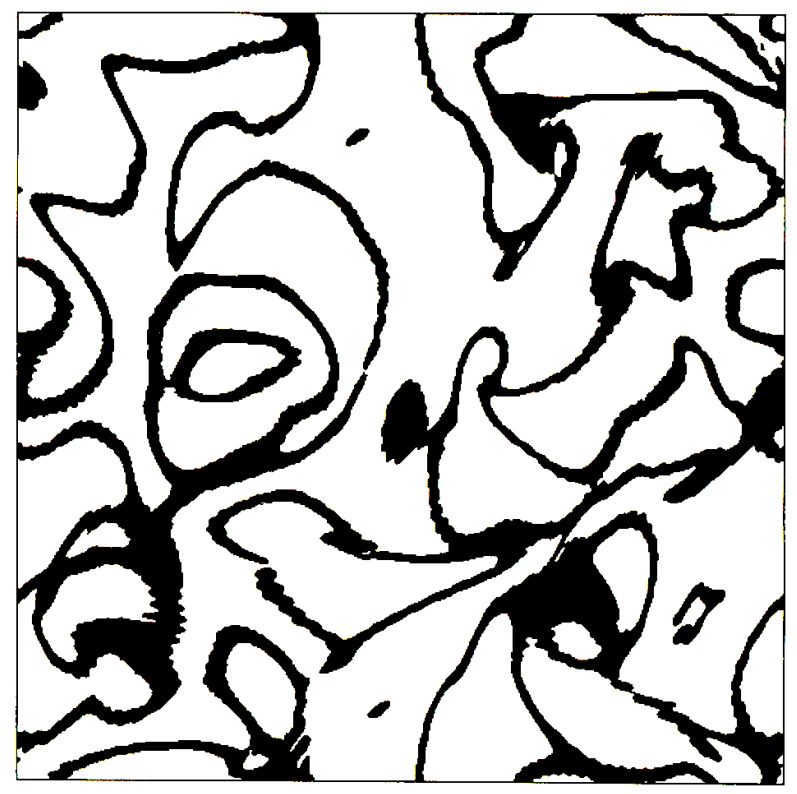

(b)
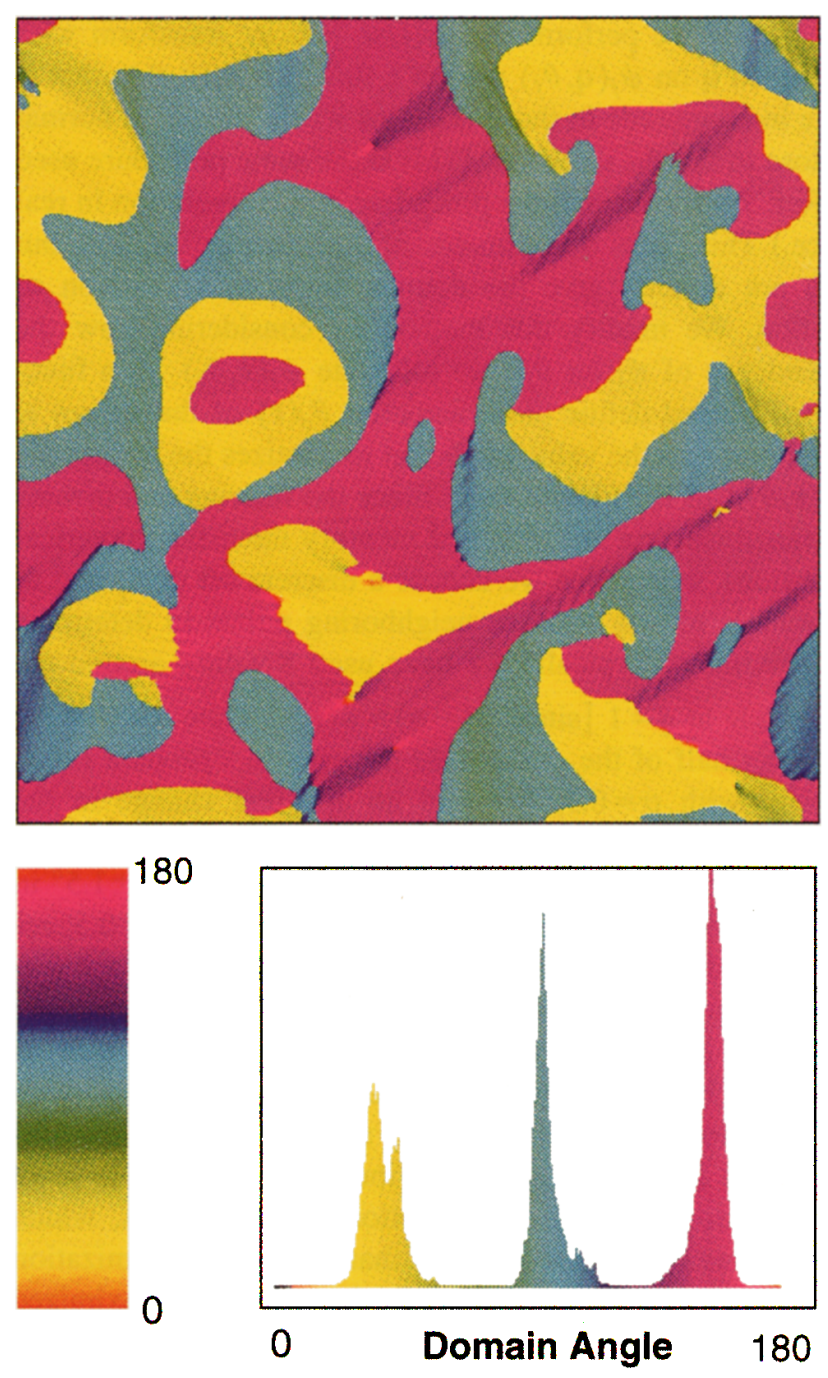

(c)

FIG. 4. Numerical extractions of the domain pattern. (a) Snapshot of stripe pattern for same parameters as in Fig. 2, except now $\epsilon=0.1$ and the system size is $120 \pi \times 120 \pi$. (b) Domain walls displayed by plotting the regions of reduced maximum amplitude of the filtered field $\psi_{f}\left(r, \theta_{d}\right.$ ) (black regions are less than $75 \%$ of the maximum amplitude). (c) Color scale plot of the domain orientation field $\theta_{d}(r)$ and a histogram of the orientations.

$a \epsilon^{1 / 2}+b \epsilon$ with $a$ and $b$ of comparable magnitudes as would be expected for such an expansion. An interesting way to test this approximate scaling of the domain structure with $\epsilon^{1 / 2}$ is to evolve the equation at two different values of the control parameter (we used $\epsilon=0.03$ and $\epsilon=0.2$ ) in system sizes scaled with $\epsilon^{-1 / 2}$. A comparison of the domain and domain wall structure on the scaled length scale $\epsilon^{1 / 2} x$ is shown in Fig. 8. The sizes of the domains and the thickness of the domain walls appear quite similar between the two sets of parameters in these scaled plots, confirming the scaling. One difference that is apparent from this comparison, and appears quite generally, is that there is more flexibility towards variations of the roll orientation within domains at the larger $\epsilon$ (compare the continuous variation of the colors within the domains in the bottom left panel with the almost uniform colors, except for distortions arising from isolated dislocations, in the top left panel). Also the structure of the domain walls appears to change from one involving the overlap of the two sets of rolls without much reorientation of the rolls (evident as the striations in the domain wall regions in the 


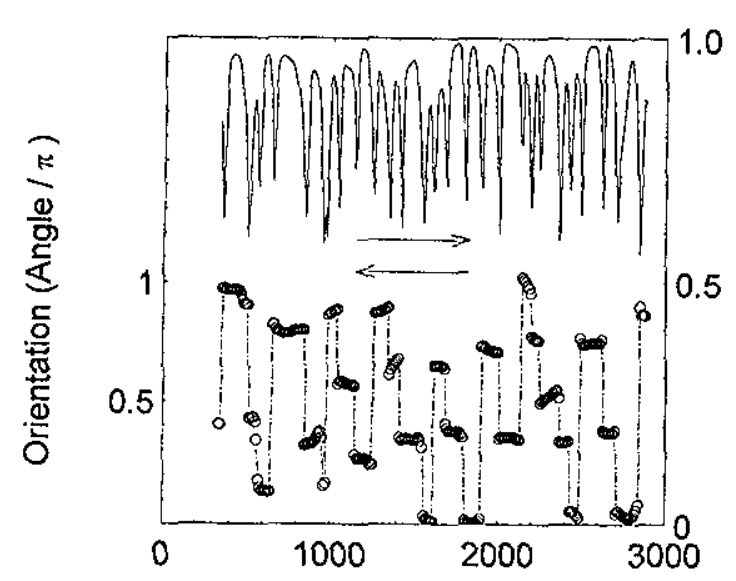

(a)

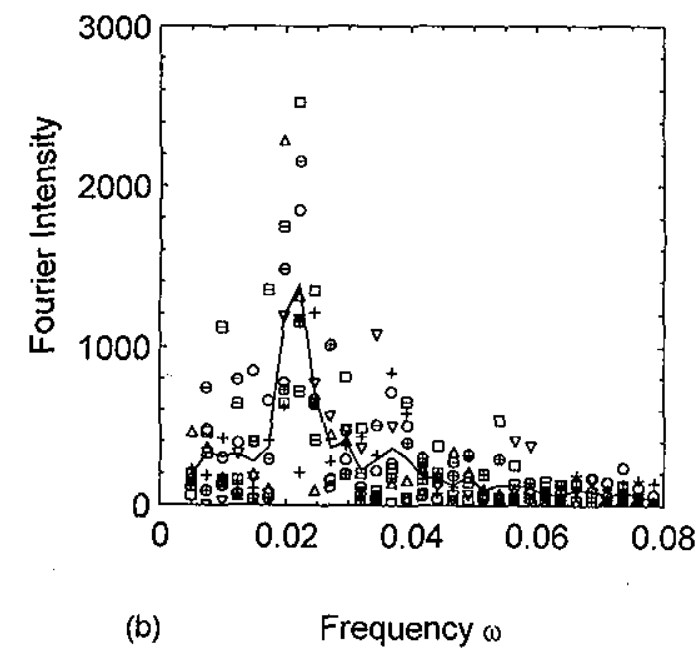

FIG. 5. (a) Time plot of the stripe orientation $\theta_{d}(\mathbf{r}, t)$ (lower trace) and maximum amplitude $\left|\psi_{f}\left(\mathbf{r}, t ; \theta_{d}\right)\right|$ (upper trace) at one point in the system for the same parameters as in Fig. 2. (b) Power spectrum of the orientation $\theta_{d}(t)$ for eight points distributed over the system (symbols) and the average (solid line) showing a broad peak from which a characteristic time scale of the motion can be identified.

top right panel), to one involving a rapid reorientation of the rolls at larger $\epsilon$ (bottom right).

The variation of the domain structure with the nonlinear coefficient $g_{3}$, or with $g_{-}=2 /\left(1+g_{3}\right)$, is shown in Fig. 7 (b). The first thing to remark is that, as was found in the amplitude equation model, the persistent chaos survives into the parameter range $g_{-}>1$ (corresponding to $g_{3}<1$ ) where the ordered stripe phase is also stable. We have evolved the system for $g_{3}=0.9$ for example (with $\epsilon=0.3$ and a system size $160 \pi$ ) for a time 16000 from an initial condition of a domain state at $g_{3}=1.1$ and find no cessation of the domain switching in this time period. This is demonstrated in Fig. 9 showing the orientation of the stripes at a particular point: the same switching phenomenon as at larger values of $g_{3}$ is evident. Correspondingly the correlation length, although growing as $g_{3}$ gets smaller, does not diverge approaching the point where the uniform stripes become stable. Bistability of the chaotic domain state and the uniform stripe state is thus an accurate prediction of the truncated amplitude equation

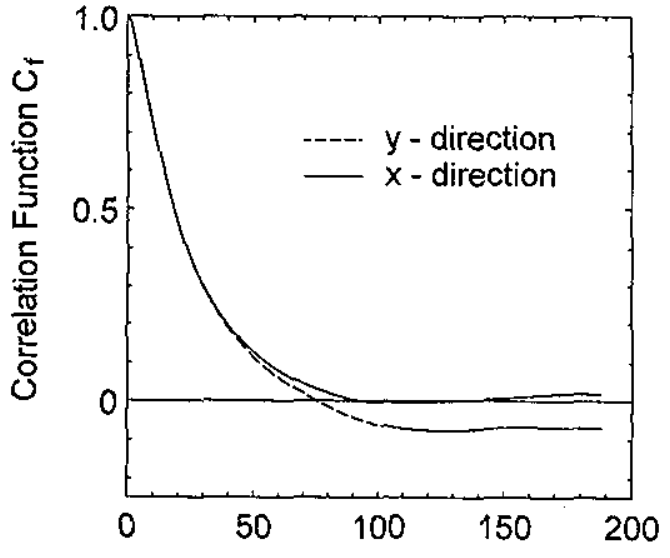

Distance $R$

FIG. 6. Filtcred correlation function $C_{\mathcal{C}}(\mathbf{R})$ as a function of separation along the $x$ and $y$ directions for the same parameters as Figs. 2 and 3 with system size $120 \pi$.

model. We cannot of course compare the quantitative value of the correlation length with the one calculated in the amplitude equation, since they are evaluated by rather different algorithms, and, since neither diverge, there is no "universal" behavior to extract. However a comparison with Fig. 2(b) of Ref. 15 shows that the qualitative trends of the variation of the correlation length with $g_{-}$are similar. Interestingly, a linear fit in the $\xi^{-1}$ vs $g_{3}$ plot would give a divergence of the correlation length at the potential point $g_{3}=0$. However the scatter in the results is too large to make this a confident result, and we might rather expect the chaotic phase to end at a saddle-node point before this extrapolation point is reached.

We can also attempt to compare the dependence of the characteristic time on $g_{3}$ with the predictions of the amplitude equation model. From Fig. 5 we found the time $\tau_{d} \simeq 300$ for $g_{3}=1.5\left(g_{-}=0.8\right), \quad \epsilon=0.3$. A corresponding plot at $g_{3}=1.1\left(g_{-}=0.95\right)$ gives a switching time $\tau_{d} \simeq 850$ showing a strong dependence of $\tau_{d}$ on $g$ - as was seen in the amplitude equation model where a ratio of around 2 was found over this range. Because of the imprecision in defining the appropriate characteristic time we have not pursued a quantitative comparison further than this.

An interesting question that we have not been able to fully answer is the question of the possible long range orientational order in these structures. The concentration of domain intensity at a discrete set of orientations may be a finite size effect that would go away as the system size increases when very distant regions of the system are effectively independent. On the other hand, the effect could persist for arbitrarily large system sizes, in which case the chaotic domain state would truly show long range orientational order, whilst still being disordered, through the existence of domains, in the translational degrees of freedom. Because of the limitation of the system sizes that are practical to simulate and possible delicate dependence on the underlying numerical mesh, we do not yet have a definitive answer to this question.

One way to address the issue of long range order is to 


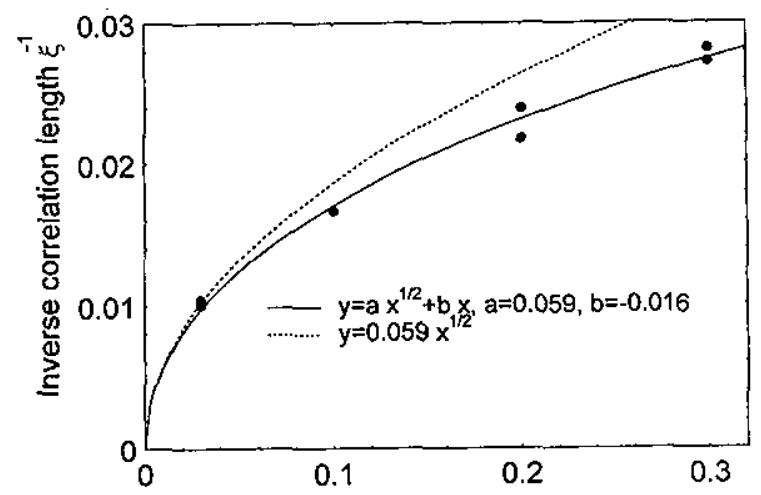

(a)

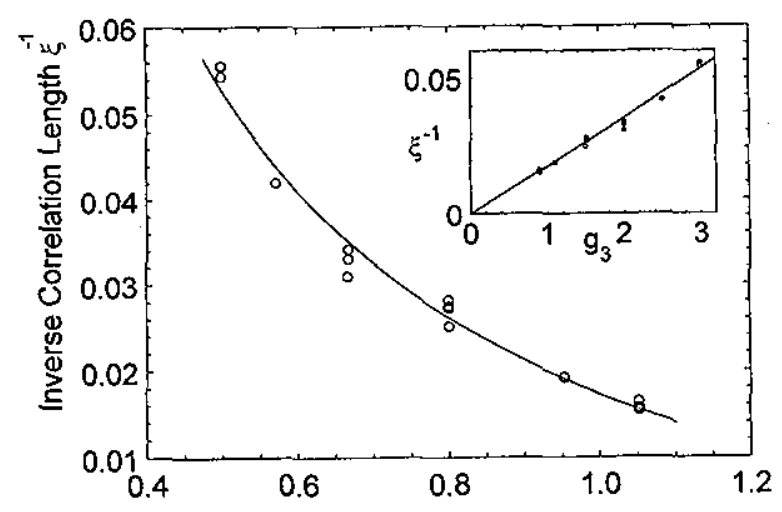

(b)

g.

FIG. 7. Dependence of the correlation length $\xi$ on the parameters for $\theta_{\mathrm{XL}}=\pi / 3$. (a) Dependence on $\epsilon$ for $g_{3}=1.5$ (solid circles) and fits to the expected $\sqrt{\epsilon}$ for small $\epsilon$. (b) Dependence on $g_{-}=2 /\left(1+g_{3}\right)$ for $\epsilon=0.3$ (open circles). Inset plots the same data directly against $g_{3}$ and shows a linear fit to the dependence on $g_{3}$. In these figures the different symbols at the same parameter values are from different time segments of the same run, or from different system sizes or mesh resolutions used to test the convergence and scatter of the results.

look at the appropriate correlation function that would show the expected long range order. If the order is in the orientation of domains aligned at multiples of $\pi / 3$, plus some offset, then an appropriate correlation function to look at is

$$
C_{6}(\mathbf{R})=<\cos 6\left[\theta_{d}(\mathbf{r}+\mathbf{R}, t)-\theta_{d}(\mathbf{r}, t)\right]>_{\mathbf{r}, t} .
$$

In the presence of the long range order this function should approach a nonzero constant as $R \rightarrow \infty$. On the other hand the function

$$
C_{2}(\mathbf{R})=<\cos 2\left[\theta_{d}(\mathbf{r}+\mathbf{R}, t)-\theta_{d}(\mathbf{r}, t)\right]>_{r, t}
$$

would asymptote to zero at large separations.

We show these quantities in Fig. 10 for parameters $g_{3}=1.5\left(g_{-}=0.8\right)$, and for two values of $\epsilon, 0.2$ and 0.3 . In both cases $C_{2}(r)$ appears to tend to zero at large separations [we do not believe the small anticorrelation in $C_{2}(r)$ at large separations is significant], whereas $C_{6}(r)$ remains larger, with a trend that appears to be consistent with a nonzero asymptotic value, although certainly other dependencies (e.g. power law decay) cannot be ruled out. The asymptotic constant would then have a strong $\epsilon$ dependence. We do not present these as conclusive results, because of the reasons mentioned above, and also because we have not investigated the dependence on the history of the runs leading to these figures and have not repeated the calculation over a number of initial conditions.

\section{Qualitative behavior at other parameter values}

\section{Dependence on $g_{2}, g_{3}$}

As the strength of the symmetry breaking term $\left|g_{2}\right|$ increases (with the associated change in $g_{3}$ to keep the maximum growth rate at $\pi / 3$ ) the patterns become more disordered, Fig. 11(a), with clear domains of one set of parallel rolls less discernible. Instead in some regions the local structure takes on a more cellular nature, appearing to be the superposition of different stripe states. These cellular domains are interspersed with domains that remain better described as stripes. A histogram of the stripe orientation $\theta_{d}$, Fig. 11(b), calculated in the same way as above shows a much more uniform distribution, without the clumping around three orientations found at smaller values of $g_{3}$. The Fourier-intensity versus time plots analogous to Fig. 3(a) are also more highly disordered. The switching angle extracted from a correlation plot analogous to Fig. 3(b) is larger than $\pi / 3$, and particularly for smaller values of $\epsilon$ moves closer to $\pi / 2$. We do not have an explanation for this latter result.

\section{Dependence on $g_{2} / g_{3}$}

An interesting question is how sensitive the behavior is to the angle at which the maximum growth rate occurs: for angles away from a rational fraction of $\pi$ a truncated mode amplitude description would not be possible. As an example we have investigated this question by using $g_{2} / g_{3}$ to tune the initial instability and maximum growth rate to $51^{\circ}$. The resulting behavior we find is visually little changed from the behavior at a rational switching angle, with the same domain switching phenomenon apparent in the dynamics. This is also demonstrated by the time dependence of the orientation at a single point shown in Fig. 12(a) for the parameters $g_{2}=-2.46, g_{3}=0.522$ (corresponding to $g_{-}=0.67$ ) and $\epsilon=0.3$. [The stability curve for these parameters is shown in Fig. 1(b).] However, as is evident in Fig. 12(a), although each switching event is quite analogous to that shown in Fig. 6 , there is now a long term drift of the local orientation as the deficit of the switching angle from $\pi / 3$ accumulates. This is also apparent from the correlation plot analogous to Fig. 3(b) that we do not show: the plot is similar, except the first peak in the correlation occurs at an angle away from $\pi / 3$ (we find about $54^{\circ}$ ) and subsequent peaks occur at multiples of this angle. The angle correlation functions are shown in Fig. 12(b): we see that $C_{6}(r)$ is now less than $C_{2}(r)$ for all $r$, and the curves are consistent with zero large distance asymptotic values, suggesting no long range order, at least for these order parameters, in this case. 


\section{$\varepsilon=0.03, \Gamma=180 \pi$}
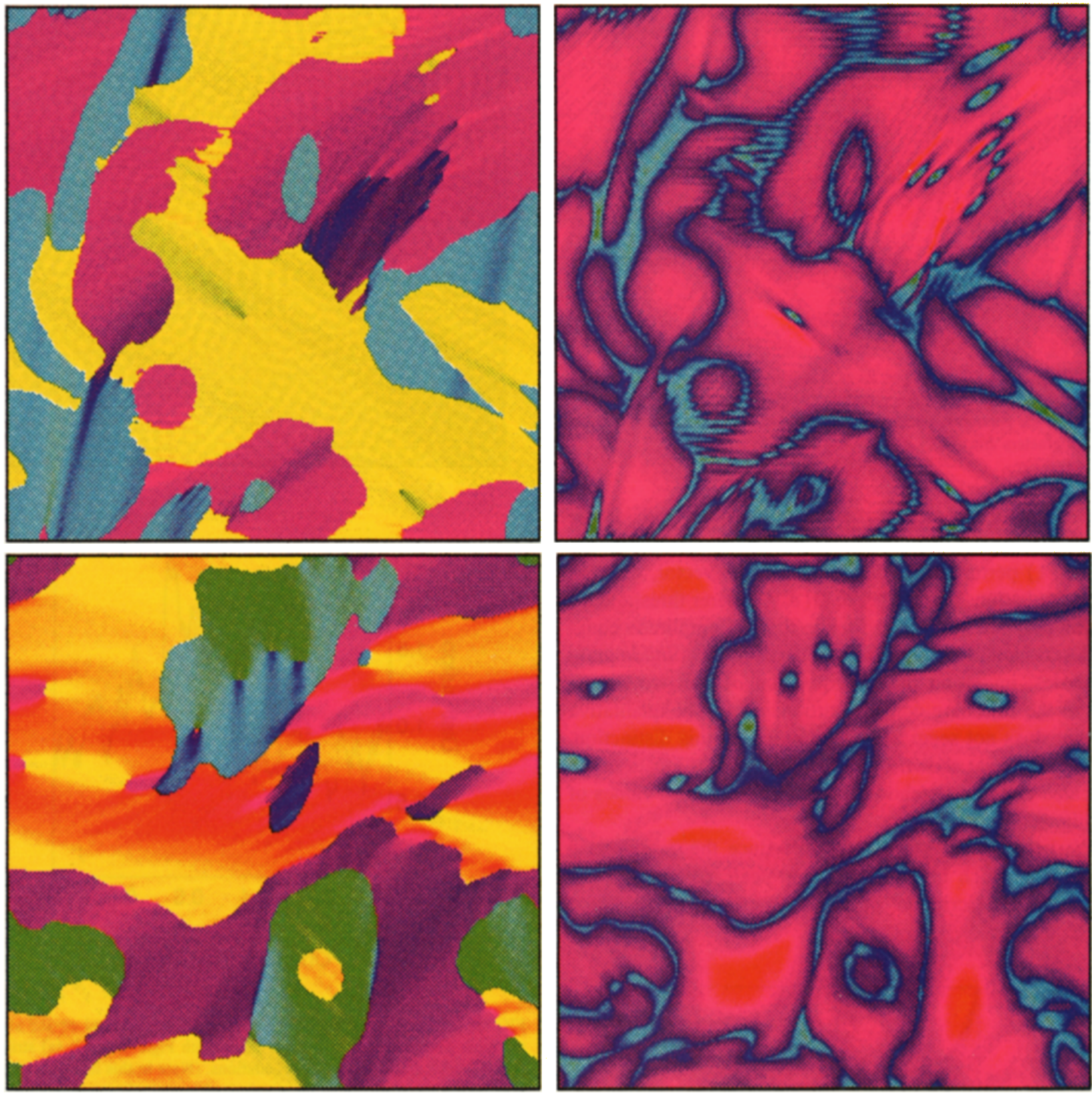

\section{$\varepsilon=0.2, \Gamma=62 \pi$}

FIG. 8. Scaling of the domain patterns with $\sqrt{\epsilon}$. The top two panels show the orientation field $\theta_{d}(\mathbf{r})$ (left) and maximum magnitude $\left|\psi_{f}\left(\mathbf{r}, \theta_{d}\right)\right|($ (right) for $\epsilon=0.03$ in a system of size $\Gamma=180 \pi$, and the lower two panels for $\epsilon=0.2$ and a system size scaled with $\sqrt{\epsilon}$, i.e., $\Gamma \simeq 62 \pi$. The similarity of the two sets of scaled patterns demonstrates in a qualitative way the anticipated scaling of lengths with $\sqrt{\epsilon}$. Some differences between the two sets are pointed out in the text.

\section{v. CONCLUSIONS}

The numerical simulations of the Swift-Hohenberg equation modified to take into account the broken chiral symmetry induced by rotation confirms the essential results of the three mode truncated amplitude equation description of rotating convection. We find a similar domain switching phenomenon, and the main quantitative analyses of the spatial structure and dynamics there are reproduced. The scaling of the lengths with $\epsilon^{-1 / 2}$ predicted by the amplitude equation are approximately confirmed. The much richer range of possibilities in the dynamics of Eq. (3), such as a continuum range of stripe orientations, does not seem to affect the major features of the patterns or dynamics. It is interesting to note that at larger values of $\epsilon$ the possibility of phase grain boundaries occurs in Eq. (3) and is seen in the numerical structures. In these the stripe angle changes by a large amount over the 


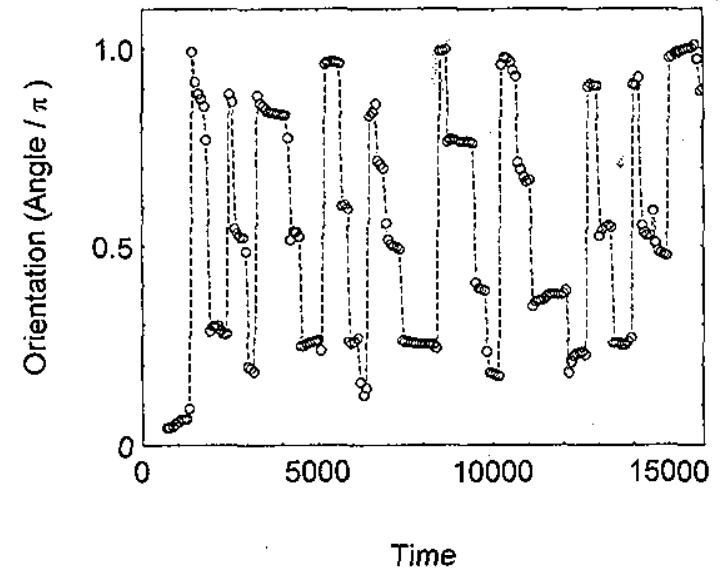

FIG. 9. Orientation of the stripes $\theta_{d}$ as a function of time at a single point in a system of size $160 \pi$ demonstrating the persistence of the switching dynamics in the parameter range where a single set of stripes is also a stable state. (Parameters are $\epsilon=0.3, g_{3}=0.9, g_{2}=-1.56$.)

relatively small distance of the thickness of the wall. Phase grain boundaries cannot occur at smaller $\epsilon$, since they involve $O(1)$ perturbations of the local wave vector, whereas such perturbations are limited to $O\left(\epsilon^{1 / 2}\right)$ nearer threshold. In addition they are not allowed for in the real amplitude equation description of 'Tu and Cross; where only amplitude grain boundaries, involving the superposition of the two amplitudes of the adjoining domains, are included. The qualitative behavior does not seem to depend much on this distinction, as is perhaps best shown by the similarity of the patterns in the scaled coordinates shown for the two different values of $\epsilon$ in Fig. 8 .

We have found useful ways to reduce the complicated field $\psi(\mathbf{r}, t)$ which involves the short scale structure of the stripes, to a description of the domain orientation field

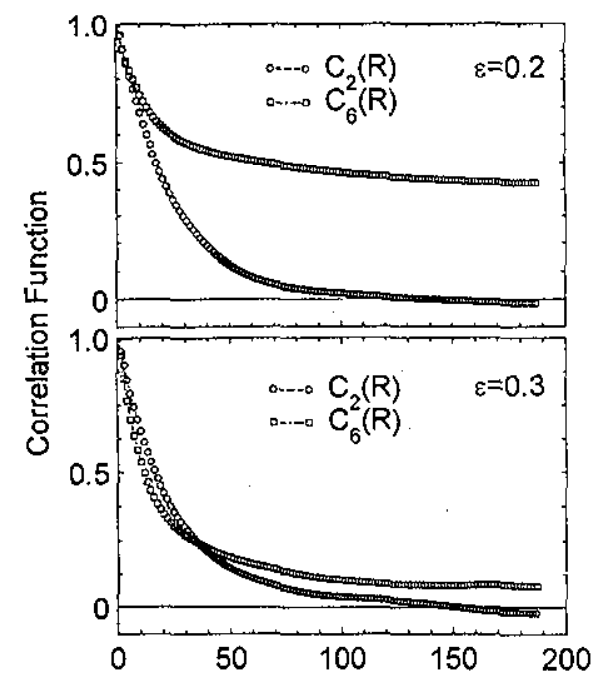

Distance $\mathrm{R}$

FIG. 10. Correlation functions $C_{2}$ and $C_{6}$ defined in Eqs. (25) and (26) as a function of distance $\mathbf{R}$ for $g_{3}=1.5, g_{2}=-2.60$, and for two values of $\epsilon=0.2$, and 0.3 . in a system size $80 \pi$.

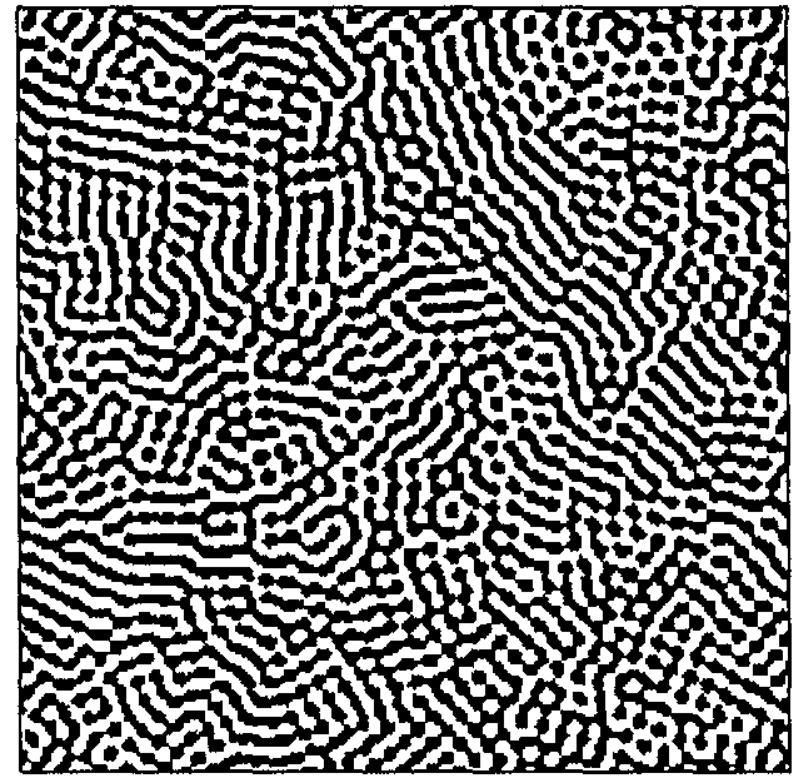

(a)

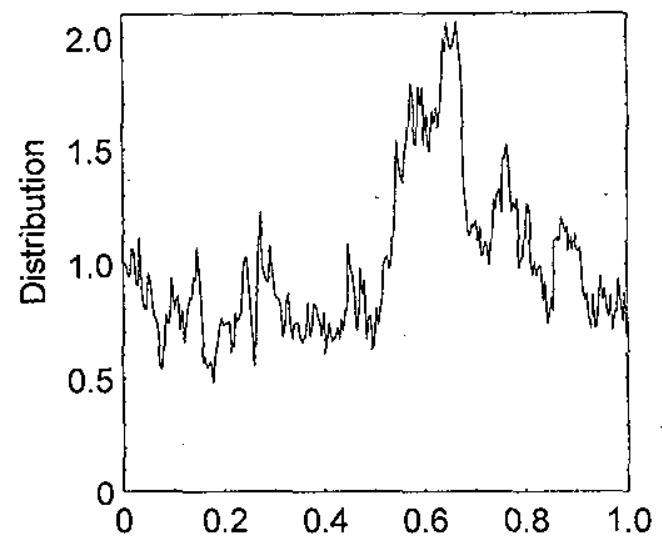

(b) Orientation (Angle $/ \pi$ )

FIG. 11. Domain pattern at a larger value of $g_{3}=3.0$ and $\epsilon=0.3$ showing the more disordered state in comparison with Figs. 2 and 3: (a) stripe pattern; (b) histogram of domain orientations. (Note in this histogram we have divided out the response of the maximization procedure, using 12 windows of width 0.5 , to a uniform distribution to reduce the spurious peaks.)

$\theta_{d}(\mathbf{r}, t)$, and we used this reduced field to extract correlation lengths, power spectra and to investigate, tentatively, questions of long range order. In the future we would like to study the application of algorithms for characterizing the chaotic dynamics, such as dimensions or Lyapunov exponents, to this description. Such algorithms may be useful in other situations with dynamic domains, such as the transient domain coarsening in the potential Swift-Hohenberg equation and other models that do not show persistent dynamics.

Finally, we suggest that the chaotic domain state in rotating convection provides a fascinating example of spatiotemporal chaos worthy of further theoretical and experimental investigation. One particular area to be pursued is the possibility of phase transitions to states with long range or 

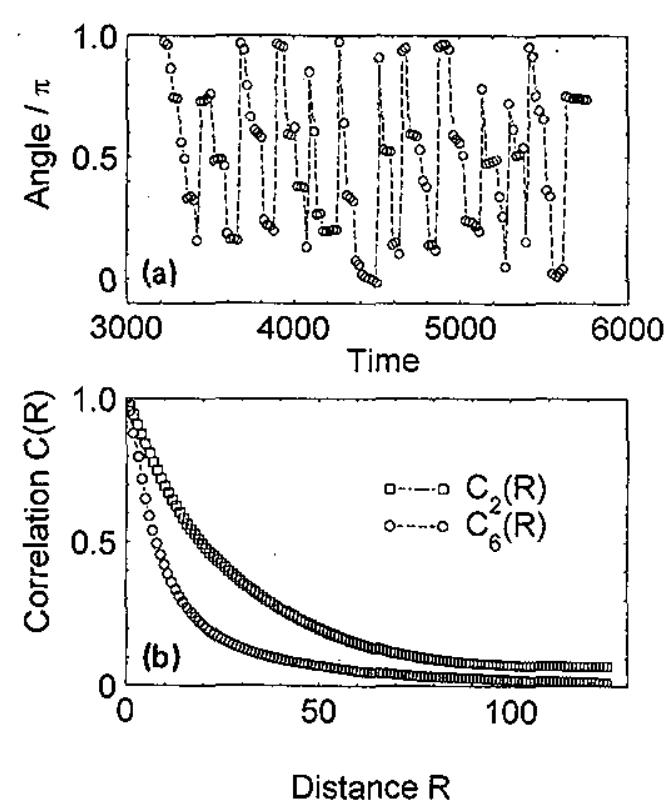

FIG. 12. Behavior at a "nonrational" angle: (a) stripe orientation at a fixed point as a function of time; and (b) spatial correlation functions defined in Eqs. (25) and (26); for $\theta_{\mathrm{KL}}=51^{\circ}\left(g_{2}=-2.46, g_{3}=0.522\right.$, corresponding to $g_{-}=0.67$, and $\epsilon=0.3$ ).

power law orientational order, and the question of how such phase transitions compare with analogous phase transitions in equilibrium systems.

\section{ACKNOWLEDGMENTS}

This work was supported by the National Science Foundation through Grant No. DMR-9013984, by the U.S. De- partment of Energy through Grant No. DE-FG0389ER25073, and by a generous award of computer time from the San Diego Supercomputer Center. Yuhai Tu would like to acknowledge support by Caltech through a Division Postdoctoral Fellowship.

${ }^{1}$ S. W. Morris, E. Bodenshatz, D. S. Cannell, and G. Ahlers, Phys. Rev. Lett. 71, 2026 (1991).

${ }^{2}$ H. Xi, J. D. Gunton, and J. Vinals, Phys. Rev. E 47, 2987 (1993).

${ }^{3}$ S. Nasuno, S. Takeuchi, and Y. Sawada, Phys. Rev. A 40, 3457 (1989).

${ }^{4}$ E. S. Braun, S. Rasenat, and V. Steinberg, Europhys. Lett. 15, 597 (1991).

${ }^{5}$ H. R. Brand, P. S. Lomdahl, and A. C. Newell, Physica 23, 345 (1986).

${ }^{6}$ P. Coullet and J. Lega, Europhys. Lett. 7, 511 (1988).

${ }^{7}$ Q. Ouyang and H. L. Swinney, Chaos 1, 411 (1991).

${ }^{8}$ M. Seul, L. R. Monar, L. Ogorman, and R. Wolfle, Science 254, 1616 (1991).

${ }^{9}$ R. E. Rosensweig, M. Zahn, and R. J. Shumovich, J. Magn. Magnetic Mat. 39, 127 (1983).

${ }^{10} \mathrm{~J}$. Vinals, E. Hernandez-Garcia, M. San-Miguel, and R. Toral, Phys. Rev. A 44, 1123 (1991).

${ }^{11}$ G. Kuppers and D. Lortz, J. Fluid Mech. 35, 609 (1969).

${ }^{12} \mathrm{~F}$. H. Busse and F. H. Heikes, Science 208, 173 (1980).

${ }^{13}$ F. H. Heikes and F. H. Busse, Ann. N.Y. Acad. Sci. 357, 28 (1980).

${ }^{14}$ E. Bodenschatz, D. S. Cannell, R. Ecke, Y. Hu, K. Lerman, and G. Ahlers, Physica D 61, 77 (1992).

${ }^{15} \mathrm{Y}$. Tu and M. C. Cross, Phys. Rev, Lett. 69, 2515 (1992).

${ }^{16}$ M. C. Cross and P. C. Hohenberg, Rev. Mod. Phys. 65, 851 (1993).

${ }^{17}$ J. B. Swift and P. C. Hohenberg, Phys. Rev. A 15, 319 (1977).

${ }^{18}$ E. Kuo and M. C. Cross, Phys. Rev. E 47, 2245 (1993).

${ }^{19}$ C. Canuto, M. Hussaini, A. Quarteroni, and T. Zang, Spectral Methods in Fluid Dynamics (Springer, Now York, 1988).

${ }^{20}$ R. A. Friesner, L. S. Tuckerman, B. C. Dornblaser, and T. V. Russo, J. Sci. Comput. 44, 327 (1989).

${ }^{21}$ W. H. Press, S. A. Teukolsky, W. T. Vetterling, and B. P. Flannery, Numerical Recipes, 2nd ed. (Cambridge University Press, Cambridge, 1992), Chap. 16.

${ }^{22}$ F. Zhong and R. E. Ecke, Chaos 2, 163 (1992).

${ }^{23}$ L. Ning and R. E. Ecke, Phys. Rev. E 47, 2991 (1993).

${ }^{24}$ A. Bovick, IEEE Trans. Inf. Theory 38, 691 (1992). 